
\title{
R Reserach S Suare \\ Prediction of Clostridium difficile infection in patients with polymerase chain reaction using a classification and regression tree
}

Diana Martínez-Ruíz

Fundación Valle del Lili

Luis Gabriel Parra-Lara

Fundación Valle del Lili https://orcid.org/0000-0002-4877-1117

Fernando Rosso ( $\nabla$ fernando.rosso@fvl.org.co )

Fundación Valle del Lili

Research article

Keywords: Clostridium difficile,Polymerase chain reaction,Decision trees,Diagnosis

Posted Date: January 13th, 2020

DOl: https://doi.org/10.21203/rs.2.20682/v1

License: (c) (i) This work is licensed under a Creative Commons Attribution 4.0 International License.

Read Full License 


\section{Abstract}

\section{Background}

Clostridium difficile infection (CDI) is commonly diagnosed with the polymerase chain reaction (PCR), but this test finds a high percentage of false positives, so their use and interpretation in CDI is a challenge in the clinical practice. That is why it is necessary to define an algorithm to optimize the use of PCR that considers clinical characteristics to classify patients with diarrhea as CDI or without CDI.

\section{Objective}

To identify a predictive algorithm with the clinical features that best classify patients with CDI vs. without $\mathrm{CDI}$, to help physicians in making decisions to request PCR.

\section{Materials and methods}

A case-control study was conducted at Fundación Valle del Lili. The population was inpatients between 2012 and 2016, with 18 or more years, and diarrhea, abdominal pain, or other nonspecific gastrointestinal symptoms who underwent PCR. Cases were defined as patients with positive PCR for $C$. difficile and as controls patients with negative PCR for $C$. difficile. Predictive algorithms to classify patients was constructed using a classification tree, classification and regression tree (CART).

\section{Results}

A total of 149 patients were included (48 cases with positive PCR and 99 controls with negative PCR). The CART has a high capacity to classify patients with a negative PCR correctly. It includes variables about the history of antibiotics use, the use of proton-pump inhibitor, the use of ranitidine, and the use of antifungal drugs. The CART showed sensitivity $64.6 \%$, specificity $85.8 \%$, positive predictive value $68.8 \%$, negative predictive value $83.3 \%$. and AUC $79.7 \%$.

\section{Discussion}

CART had good specificity and a high negative predictive value; it could be considered as an algorithm to identify conditions that indicate when it is not necessary to perform a PCR test in a patient symptom of CDI.

\section{Introduction}

Clostridium difficile infection (CDI) is the most common cause of healthcare-associated diarrhea(1). The risk factors identified for CDI are the use of antibiotics, stomach acid-suppressing medications, primarily proton-pump inhibitors (PPI), advanced age (above 64 years), prior hospitalization, exposure to CDI patients, weak health condition, among others(2). 
In the past two decades, the incidence and severity of CDI have increased worldwide. Several reports have shown that $C$. difficile affects 20 to $50 \%$ of patients in the hospital environment. Indeed, a recent metaanalysis reported that $C$. difficile accounted for $20 \%$ of all antibiotic-.associated diarrhea cases among hospitalized patients $(3,4)$. Although there is a lack of data about this issue from Latin America, a Colombian study found that $16.7 \%$ of gastrointestinal infections in hospitalized adults were caused by $C$. difficile(5).

$\mathrm{CDI}$ is commonly diagnosed with the polymerase chain reaction (PCR), which is specific for genes encoding toxins produced by $C$. difficile(6,7). Although this test is useful in clinical practice, its interpretation and applicability are challenging(7). Approximately $50 \%$ of hospitalized patients may be asymptomatically colonized by toxigenic $C$. difficile(7). Moreover, a positive PCR result does not distinguish between active toxin production and asymptomatic carriage, which results in a large number of false positives $(7,8)$. All these issues lead to increased costs, unnecessary isolation, and antibiotic overuse(9).

One of the most significant challenges in the management of patients with CDI associated diarrhea is to underpin the advance of diagnostic decision-making algorithms in the clinical practice scenario. Clinical algorithms can increase the pretest likelihood of CDI diagnosis. Furthermore, the use of such models in daily clinical practice may lower the overuse of diagnostic tests, thus reducing costs and improving the correct utilization of specific treatments for CDI. The present study used a classification tree to develop a predictive model for CDI diagnosis using a PCR test among inpatients attended at Cali, Colombia.

\section{Materials And Methods}

\section{Data source}

We performed a retrospective case-control study from 2012 to 2016 at Fundación Valle del Lili (FVL) in Cali, Colombia. FVL is a level IV university hospital affiliated with Universidad Icesi and serves as a referral facility for the southwest region of Colombia. All data were obtained directly from the clinical records.

\section{Patients and Controls}

We included hospitalized patients older than 18 years old with diarrhea, abdominal pain, or other nonspecific gastrointestinal symptoms and that underwent PCR testing for $C$. difficile. Cases were defined as patients with a positive PCR result for $C$. difficile. Controls were defined as patients with negative PCR results.

\section{Laboratory methods}

The FVL clinical practice guideline (CPG) for CDI diagnosis include PCR test. A Xpert ${ }^{\circledR}$ C. difficile assay (Xpert CD assay; Cepheid, Sunnyvale, CA, USA) is a multiplex real-time PCR assay that uses primers targeted to the cytotoxin gene $(t c d B)$, binary toxin genes ( $c d t A$ and $c d t B$ ), and a single nucleotide deletion 
at position 117 in the $t c d C$ gene. As a result, the Xpert $C D$ assay can detect toxigenic $C$. difficile strains and differentiate $C$. difficile presumptive 027/NAP1/BI(10).

\section{Statistical analysis}

Predictive algorithms to classify patients with $\mathrm{CDI}$ and without $\mathrm{CDI}$ were designed using a classification and regression tree (CART). There is a non-parametric technique that determines a set of logical conditions (variables) to perform binary classification of cases in homogeneous subgroups and at the same time, considers the complexity of the relationships between the independent variables. For this study, the CART explains why patients are classified as negative or positive PCR, achieving a low rate of misclassification $(11,12)$.

In each division (node), the tree selects the independent variable that best classifies patients with CDI or without CDI. Then, the algorithm includes variables until achieving the highest possible homogeneity or few observations. The steps for developing the CART were: 1) Construction of the maximum tree with variables that showed significantly less or equal to $20 \%$ in the bivariate analysis. For the bivariate analysis, quantitative data were compared using the Mann-Whitney $\mathrm{U}$ test, and qualitative data using the Chi-Squared test or Fisher's exact test; 2) The maximum tree was built using the Gini index, which was a criterion to determine the best division. The minimum change in the rate of misclassification was a criterion for stopping the tree (adding new variables); 3) Some ten patients were established as the minimum number of observations per division; 4) The process of pruning backward, to obtain a parsimonious tree, was done with cross-validation using the same sample, and the cost-complexity mean was zero. The resulting classification tree was evaluated for discriminative capacity through sensitivity, specificity, positive and negative predictive values, overall value, likelihood ratio, and the degree of diagnostic accuracy by calculating the area under the ROC curve. All analyses were performed using software R v.3.16(13).

\section{Results}

A total of 149 patients were included in the study after fulfilled criteria, forty-eight cases with positive PCR, and ninety-nine controls with negative PCR. Two cases were excluded for the analysis.

The median age was 55 years (IQR=71-35 years). The majority were women. The most frequent comorbidity was cardiovascular disease. The leading cause of hospitalization was for non-surgical conditions $(40,9 \%, n=61)$. Table 1 shows the main clinical characteristics.

Table 2 shows the drugs prescribed to the patients. A total of $62.6 \%$ of patients had a history of antibiotics use, mainly meropenem and antifungal drugs. Other frequently used drugs were omeprazole, immunosuppressants, and steroids. Statistically significant differences were found in the use of meropenem, clindamycin, polymyxin B, antifungals, steroids, and chemotherapy. Also, regarding the history of drug use, antibiotics, PPI and $\mathrm{H} 2$ antagonists, statistically significant differences were found. 
The CART is presented in Figure 1. The CART had adequate discrimination capacity to distinguish between positive and negative PCR, only with the history of antibiotic use. However, the CART also included other variables, such as the use of PPI, the use of ranitidine, and the use of antifungal drugs, because these variables help make a better decision. The CART showed the following performance: sensitivity $64.6 \%$, specificity $85.8 \%$, positive predictive value $68.8 \%$, negative predictive value $83.3 \%$, positive likelihood ratio 4.56 , negative likelihood ratio 0.41 , and AUC $79.7 \%$ (Figure 2).

The algorithm to identify patients with negative PCR was: if a patient did not have a history of antibiotic use, the probability was $89.1 \%$. On the contrary, if a patient had a history of antibiotic use, the probability was $54.3 \%$. But if a patient had a history of antibiotic use, did not use PPI and ranitidine, the probability of a negative PCR was $78.8 \%$. Finally, if a patient had a history of antibiotic use, and did not use PPI but received ranitidine and did not use antifungal drugs, the probability of a negative PCR was $71,4 \%$.

\section{Discussion}

In low and middle-income countries, there is a lack of evidence-based diagnostic algorithms for early CDI diagnosis using a PCR test. In 2011, a study showed that the prevalence of in-hospital C. difficile was 10 per 10.000 inpatients in Cali, indicating a high prevalence of this infection and the need to identified better strategies for the control of $C$. difficile(14). Our study shows a straightforward algorithm that includes clinically relevant variables and may help clinicians in the diagnostic process of patients with suspected CDI. The CDI is an important cause of nosocomial infection, so the implementation of diagnostic strategies, such as decision trees, is an important alternative to guide physicians in making decisions.

Our CART showed that if a patient had a history of antibiotic use, the probability of a positive PCR was $45.7 \%$. Previous studies in this matter showed almost all antibiotics can increase vulnerability to CDI, but cephalosporins, fluoroquinolones, clindamycin, and certain penicillins (e.g., amoxicillin/clavulanic acid) increase risk to the greatest extent $(8,14,15)$. Above supports the results of this study regarding consider this exposure in the decision to perform a PCR.

Also, PPI has been associated with increased odds of CDI and recurrence(8). The 2018 meta-analysis by Oshima et al.(16) concluded that PPI use was associated with CDI in adult (OR 2.30, 95\% Cl 1.89-2.80; $p<0.00001)$ and pediatric patients (OR 3.00,95\% Cl 1.44-6.23; $p<0.00001$ ), and with recurrent CDI (OR $1.73,95 \% \mathrm{Cl} 1.39-2.15 ; \mathrm{p}=0.02)$. In the CART, PCR performance in patients with a history of use of PPI was $75 \%$. This finding suggests that in a case with a history of antibiotic and PPI use, it could be not necessary to use the PCR test for CDI diagnostic, and the physician will be a big probability to have a positive case of CDI without another diagnostic test.

From our results, we can recommend considering the PCR test when the patients have been exposed to $\mathrm{PPI}$, ranitidine, and antifungal drugs. Instead, if the patient has not a history of antibiotics use, the probability of finding a negative result is higher. This model demonstrated a good capacity to classify healthy patients as healthy (specificity) and a high negative predictive value and thus can be considered 
as an algorithm to identify conditions that indicate when it is not necessary to perform a PCR test in a patient with symptoms of CDI.

CDI increases patient healthcare costs due to extended hospitalization, re-hospitalization, laboratory tests, and medications. A systematic review found that CDI to be a significant economic healthcare burden in their respective settings, with an increased length of stay and costs(17). That is why our model emerges as a diagnostic alternative for middle and low-income countries, which allows optimizing the indication of PCR for the diagnosis of CDI and thus reduces the economic burden of the disease in health systems.

The CART of this study has the advantage that it is easy to interpret and implement for health workers in their daily practice because the data that contain it is available by performing a routine clinical review to the inpatients. This methodology compared other statistical methods such as regression, which has a good capacity to discriminate, allows identifying directly from the tree, the interactions between the data and the probability of having a PCR with a positive or negative result. Therefore, this tree can be a tool for the decision to perform a PCR in patients with symptoms of CDI in environments where there are restrictions to perform diagnostic tests as PCR.

Limitations. Data were collected retrospectively, which can lead to an information bias due to the absence of data in some variables. The predictive capacity of the variable "history of antibiotics use" is limited by the time of exposure, because the time from the use of antibiotics to the development of the symptoms was not collected. On the other hand, data about the use of certain antibiotics were collected, but there are unidentified if they were administered before or after the PCR. The results of this study can be only generalized to patients treated in a high complexity referral hospital with similar demographics and clinical characteristics of our research. Despite these limitations, the designed algorithm can be a valuable tool in medium and low-income countries where resources and diagnostic tests are routinely limited.

\section{Conclusions}

This CART offers a fast algorithm for deciding to perform a PCR test on patients with symptoms of CDI in a referral health facility in southwestern Colombia. Patients with a low probability of a positive PCR result are those without a history of antibiotics use. Still, if they have been exposed, the use of PPI, ranitidine, and antifungal drugs should be considered, other tests should be performed to rule out CDI.

\section{Abbreviations}

AUC: area under curve

CART: classification and regression tree

CDI: Clostridium difficile infection 
FVL: Fundación Valle del Lili

IQR: interquartile range

IRB: Institutional review board

PCR: polymerase chain reaction

PPI: proton-pump inhibitors

\section{Declarations}

\section{Ethics approval and consent to participate}

All procedures performed in studies involving human participants were in accordance with the ethical standards of the institutional research committee and with the 1964 Helsinki Declaration and its later amendments or comparable ethical standards. The Institutional Review Board - Comité de Ética en Investigación Biomédica approved the study protocol (Protocol Number: 554).

\section{Consent for publication}

Not applicable

Availability of data and materials

Data is property of authors and could be available by request at fernando.rosso@fvl.org.co

\section{Competing interests}

The authors declare that they have no competing interests

\section{Funding}

No funding. This article received no specific grant from any funding agency in the public, commercial, or non-for-profit sectors.

\section{Authors' contributions}

DMM, LGPL, and FR contributed equally to this work. DMM and LGPL designed the research. All authors performed the research, analyzed the data, and wrote the paper. All authors made important intellectual contributions to the manuscript, and all authors approved the final version before submission. FR supervised all the process.

\section{Acknowledgements}


The authors would like to thank Jorge Cedano MD, Aida Manuela Galvis MD, Stephania González MD, Carlos Vélez MD, Juan Sebastián Solarte MD, María Paula Hormaza MD for their collaboration in the data collection process. Also, to Centro de Investigaciones Clínicas - Fundación Valle del Lili for their constant support.

\section{References}

1. Cohen SH, Gerding DN, Johnson S, Kelly CP, Loo VG, McDonald LC, Pepin J, Wilcox MH. Clinical Practice Guidelines for Clostridium difficile Infection in Adults: 2010 Update by the Society for Healthcare Epidemiology of America (SHEA) and the Infectious Diseases Society of America (IDSA). Infect Control Hosp Epidemiol. 2010 May;31(5):431-55.

2. Chhipi-Shrestha G, Mori J, Hewage K, Sadiq R. Clostridium difficile infection incidence prediction in hospitals (CDIIPH): a predictive model based on decision tree and fuzzy techniques. Stoch Environ Res Risk Assess. 2017;31(2):417-30.

3. Burnham C-AD, Carroll KC. Diagnosis of Clostridium difficile infection: an ongoing conundrum for clinicians and for clinical laboratories. Clin Microbiol Rev. 2013 Jul;26(3):604-30.

4. Nasiri MJ, Goudarzi M, Hajikhani B, Ghazi M, Goudarzi H, Pouriran R. Clostridioides (Clostridium) difficile infection in hospitalized patients with antibiotic-associated diarrhea: A systematic review and meta-analysis. Anaerobe. 2018 Apr;50:32-7.

5. Zea JW, Salazar CL. Enfermedad asociada a Clostridium difficile: prevalencia y diagnóstico por laboratorio. Infectio. 2012;16(4):211-22.

6. Tartof SY, Yu KC, Wei R, Tseng HF, Jacobsen SJ, Rieg GK. Incidence of polymerase chain reactiondiagnosed Clostridium difficile in a large high-risk cohort, 2011-2012. Mayo Clin Proc [Internet]. 2014;89(9):1229-38. Available from: http://dx.doi.org/10.1016/j.mayocp.2014.04.027

7. Kufelnicka AM, Kirn TJ. Effective utilization of evolving methods for the laboratory diagnosis of Clostridium difficile infection. Clin Infect Dis. 2011;52(12):1451-7.

8. Mullish BH, Williams HR. Clostridium difficile infection and antibiotic-associated diarrhoea. Clin Med [Internet]. 2018;18(3):237-41. Available from: http://www.ncbi.nlm.nih.gov/pubmed/29858434

9. Mueller CJ, Castagno WJ, Duckworth L, McEver RC, Campbell HJ. Implementation of a Decision Tree to Optimize Clostridium difficile Testing. Am J Infect Control [Internet]. 2017;45(6):S8. Available from: http://dx.doi.org/10.1016/j.ajic.2017.04.270

10. Shin S, Kim M, Kim M, Lim H, Kim H, Lee K, Chong Y. Evaluation of the Xpert Clostridium difficile assay for the diagnosis of Clostridium difficile infection. Ann Lab Med [Internet]. 2012/08/13. 2012 Sep;32(5):355-8. Available from: https://www.ncbi.nlm.nih.gov/pubmed/22950071

11. StatSoft. Electronic Statistics Textbook [Internet]. StatSoft, editor. Tulsa: StatSoft; 2013. Available from: http://www.statsoft.com/textbook/

12. Breiman L. Classification and Regression Trees. Boca Raton: Routledge; 2017. 368 p. 
13. R Core Team. R: A language and environment for statistical computing [Internet]. Vienna: $R$ Foundation for Statistical Computing; 2019. Available from: www.R-project.org/

14. Oñate-Gutiérrez JM, Villegas MV, Correa A. Prevalencia y factores relacionados con la infección por Clostridium difficile en un centro hospitalario de alta complejidad en Cali (Colombia). Infectio. 2016;21(1):1-6.

15. Bartlett JG, Gerding DN. Clinical recognition and diagnosis of Clostridium difficile infection. Clin Infect Dis. 2008 Jan;46(Suppl 1):S12-8.

16. Oshima T, Wu L, Li M, Fukui H, Watari J, Miwa H. Magnitude and direction of the association between Clostridium difficile infection and proton pump inhibitors in adults and pediatric patients: a systematic review and meta-analysis. J Gastroenterol [Internet]. 2018;53(1):84-94. Available from: https://doi.org/10.1007/s00535-017-1369-3

17. Ghantoji SS, Sail K, Lairson DR, Dupont HL. Economic healthcare costs of Clostridium difficile infection: a systematic review. J Hosp Infect [Internet]. 2010;74(4):309-18. Available from: http://dx.doi.org/10.1016/j.jhin.2009.10.016

\section{Tables}

Table 1. Clinical characteristics of patients 


\begin{tabular}{|c|c|c|c|c|c|c|c|}
\hline \multirow[t]{2}{*}{ Data } & \multicolumn{2}{|c|}{$\begin{array}{l}\text { Total, } \\
\mathrm{n}=147\end{array}$} & \multicolumn{2}{|c|}{$\mathrm{PCR}+, \mathrm{n}=48$} & \multicolumn{2}{|c|}{$\begin{array}{l}\text { PCR-, } \\
\text { n=99 }\end{array}$} & \multirow[t]{2}{*}{$\begin{array}{c}p- \\
\text { value }\end{array}$} \\
\hline & $\mathrm{n}$ & $\%$ & $\mathbf{n}$ & $\%$ & $\mathbf{n}$ & $\%$ & \\
\hline Age, median (IQR) & \multicolumn{2}{|c|}{$\begin{array}{l}55(71- \\
35)\end{array}$} & \multicolumn{2}{|c|}{$\begin{array}{l}59.5(73.5- \\
37.5)\end{array}$} & \multicolumn{2}{|c|}{$\begin{array}{l}55(71- \\
34)\end{array}$} & 0.44 \\
\hline \multicolumn{8}{|l|}{ Sex } \\
\hline Woman & 82 & 55.8 & 23 & 47.9 & 59 & 59.6 & 0.18 \\
\hline \multicolumn{8}{|l|}{ Comorbidities } \\
\hline Cardiovascular disease & 60 & 40.8 & 20 & 41.7 & 40 & 40.4 & 0.88 \\
\hline Chronic kidney disease & 35 & 23.8 & 13 & 27.1 & 22 & 22.2 & 0.52 \\
\hline Hematologic malignancy & 31 & 21.1 & 13 & 27.1 & 18 & 18.2 & 0.21 \\
\hline Diabetes & 26 & 17.7 & 7 & 14.6 & 19 & 19.2 & 0.49 \\
\hline Infections & 23 & 15.6 & 11 & 22.9 & 12 & 12.1 & 0.09 \\
\hline Non-metastatic tumor & 21 & 14.3 & 4 & 8.3 & 17 & 17.2 & 0.15 \\
\hline Liver disease & 21 & 14.3 & 7 & 14.6 & 14 & 14.1 & 0.94 \\
\hline Rheumatologic disease & 17 & 11.6 & 4 & 8.3 & 13 & 13.1 & 0.39 \\
\hline Solid organ transplant & 16 & 10.9 & 4 & 8.3 & 12 & 12.1 & 0.58 \\
\hline Endocrine disorder & 15 & 10.2 & 4 & 8.3 & 11 & 11.1 & 0.77 \\
\hline $\begin{array}{l}\text { Chronic obstructive pulmonary } \\
\text { disease }\end{array}$ & 14 & 9.5 & 2 & 4.2 & 12 & 12.1 & 0.15 \\
\hline Congestive heart failure & 11 & 7.5 & 3 & 6.3 & 8 & 8.1 & 1 \\
\hline Bone marrow transplant & 11 & 7.5 & 6 & 12.5 & 5 & 5.1 & 0.18 \\
\hline Neurological disorder & 9 & 6.1 & 3 & 6.3 & 6 & 6.1 & 1 \\
\hline HIV & 4 & 2.7 & 1 & 2.1 & 3 & 3 & 1 \\
\hline Cerebrovascular accident & 2 & 1.4 & 1 & 2.1 & 1 & 1 & 0.55 \\
\hline Diverticular disease & 2 & 1.4 & 1 & 2.1 & 1 & 1 & 0.55 \\
\hline Peptic ulcer disease & 2 & 1.4 & 1 & 2.1 & 1 & 1 & 0.55 \\
\hline Inflammatory bowel disease & 2 & 1.4 & 0 & 0 & 2 & 2 & 1 \\
\hline AIDS & 1 & 0.7 & 0 & 0 & 1 & 1 & 1 \\
\hline \multicolumn{8}{|l|}{ Cause of hospitalization } \\
\hline Medical non-infectious & 81 & 40.8 & 26 & 43.8 & 55 & 39.4 & \multirow[t]{3}{*}{0.97} \\
\hline Infectious & 44 & 29.9 & 15 & 31.3 & 29 & 29.3 & \\
\hline Surgical & 22 & 12.9 & 7 & 12.5 & 15 & 13.1 & \\
\hline ICU hospitalization & 103 & 70.1 & 36 & 75 & 67 & 67.7 & 0.36 \\
\hline \multicolumn{8}{|l|}{ Time of previous hospitalization } \\
\hline Less than a month & 80 & 54.4 & 22 & 45.8 & 58 & 58.6 & \multirow[t]{3}{*}{0.24} \\
\hline Between 1-12 months & 46 & 31.3 & 15 & 31.3 & 31 & 31.3 & \\
\hline More than 1 year & 14 & 9.5 & 7 & 14.6 & 7 & 7.1 & \\
\hline \multicolumn{8}{|l|}{ Time between hospitalization and PCR } \\
\hline$\leq 7$ days & 59 & 40.14 & 17 & 35.4 & 42 & 42.4 & \multirow[t]{2}{*}{0.416} \\
\hline$\geq 7$ days & 88 & 59.9 & 31 & 64.6 & 57 & 57.6 & \\
\hline
\end{tabular}

Table 2. Prescription of drugs in patients 


\begin{tabular}{|c|c|c|c|c|c|c|c|}
\hline \multirow[t]{2}{*}{ Data } & \multicolumn{2}{|c|}{$\begin{array}{c}\text { Total, } \\
\mathrm{n}=147\end{array}$} & \multicolumn{2}{|c|}{$\begin{array}{c}\text { PCR+, } \\
\mathrm{n}=99\end{array}$} & \multicolumn{2}{|c|}{$\begin{array}{l}\text { PCR-, } \\
\mathrm{n}=48\end{array}$} & \multirow[t]{2}{*}{$p$-value } \\
\hline & $\mathbf{n}$ & $\%$ & $\mathrm{n}$ & $\%$ & $\mathbf{n}$ & $\%$ & \\
\hline \multicolumn{8}{|l|}{ Antibacterial } \\
\hline Meropenem & 70 & 47.6 & 29 & 60.4 & 41 & 41.4 & 0.03 \\
\hline Piperacillin/Tazobactam & 46 & 31.3 & 17 & 35.4 & 29 & 29.3 & 0.45 \\
\hline Ceftriaxone & 40 & 27.2 & 10 & 20.8 & 30 & 30.3 & 0.23 \\
\hline Cefepime & 31 & 21.1 & 14 & 29.2 & 17 & 17.2 & 0.09 \\
\hline Ciprofloxacin & 28 & 19 & 8 & 16.7 & 20 & 20.2 & 0.61 \\
\hline Sulfamethoxazole/Trimethoprim & 15 & 10.2 & 3 & 6.3 & 12 & 12.1 & 0.39 \\
\hline Polymyxin B & 12 & 8.2 & 7 & 14.6 & 5 & 5.1 & 0.06 \\
\hline Amikacin & 11 & 7.5 & 3 & 6.3 & 8 & 8.1 & 1 \\
\hline Clindamycin & 9 & 6.1 & 0 & 0 & 9 & 9.1 & 0.03 \\
\hline Ampicillin/Sulbactam & 9 & 6.1 & 2 & 4.2 & 7 & 7.1 & 0.72 \\
\hline Cefazolin & 9 & 6.1 & 4 & 8.3 & 5 & 5.1 & 0.474 \\
\hline Linezolid & 7 & 4.8 & 2 & 4.2 & 5 & 5.1 & 1 \\
\hline Moxifloxacin & 5 & 3.4 & 0 & 0 & 5 & 5.1 & 0.17 \\
\hline Oxacillin & 4 & 2.7 & 1 & 2.1 & 3 & 3 & 1 \\
\hline Doripenem & 4 & 2.7 & 2 & 4.2 & 2 & 2 & 0.6 \\
\hline Doxycycline & 4 & 2.7 & 2 & 4.2 & 2 & 2 & 0.6 \\
\hline Tigecycline & 3 & 2 & 1 & 2.1 & 2 & 2 & 1 \\
\hline Amoxicillin & 3 & 2 & 2 & 4.2 & 1 & 1 & 0.25 \\
\hline Levofloxacin & 2 & 1.4 & 0 & 0 & 2 & 2 & 1 \\
\hline Clarithromycin & 2 & 1.4 & 1 & 2.1 & 1 & 1 & 0.55 \\
\hline Ertapenem & 2 & 1.4 & 2 & 4.2 & 0 & 0 & 0.11 \\
\hline Gentamicin & 2 & 1.4 & 1 & 2.1 & 1 & 1 & 0.55 \\
\hline Antifungal & 67 & 45,6 & 28 & 58,3 & 39 & 39.4 & 0.02 \\
\hline Antiviral & 36 & 24,5 & 13 & 27,1 & 23 & 23.2 & 0.47 \\
\hline Antiparasitic & 15 & 10,2 & 5 & 10,4 & 10 & 10.1 & 1 \\
\hline \multicolumn{8}{|l|}{ Other drugs } \\
\hline Omeprazole & 113 & 76.9 & 33 & 68.8 & 80 & 80.8 & 0.1 \\
\hline Ranitidine & 77 & 52.4 & 30 & 62.5 & 47 & 47.5 & 0.09 \\
\hline Immunosuppressant & 76 & 51.7 & 21 & 43.8 & 55 & 55.6 & 0.18 \\
\hline Steroid & 69 & 46.9 & 17 & 35.4 & 52 & 52.5 & 0.05 \\
\hline NSAID & 56 & 38.1 & 22 & 45.8 & 34 & 34.3 & 0.18 \\
\hline Antidepressant & 42 & 28.6 & 15 & 31.3 & 27 & 27.3 & 0.62 \\
\hline Chemotherapy & 40 & 27.2 & 18 & 37.5 & 22 & 22.2 & 0.05 \\
\hline \multicolumn{8}{|l|}{ Pharmacological history } \\
\hline Antibiotics & 92 & 62.6 & 42 & 87.5 & 50 & 50.5 & $<0.001$ \\
\hline Immunosuppressant & 48 & 32.7 & 15 & 31.3 & 33 & 33.3 & 0.77 \\
\hline Chemotherapy & 36 & 24.5 & 13 & 27.1 & 23 & 23.2 & 0.31 \\
\hline Proton-pump inhibitor & 34 & 23.1 & 18 & 37.5 & 16 & 16.2 & $<0.0001$ \\
\hline Corticosteroids & 33 & 22.4 & 11 & 22.9 & 22 & 22.2 & 0.25 \\
\hline NSAID & 10 & 6.8 & 1 & 2.1 & 9 & 9.1 & 0.45 \\
\hline $\mathrm{H} 2$ antagonist & 10 & 6.8 & 8 & 16.7 & 2 & 2 & $<0.0001$ \\
\hline Antidepressant & 7 & 4.8 & 4 & 8.3 & 3 & 3 & 0.06 \\
\hline Laxatives & 4 & 2.7 & 2 & 4.2 & 2 & 2 & 0.23 \\
\hline
\end{tabular}


Figures

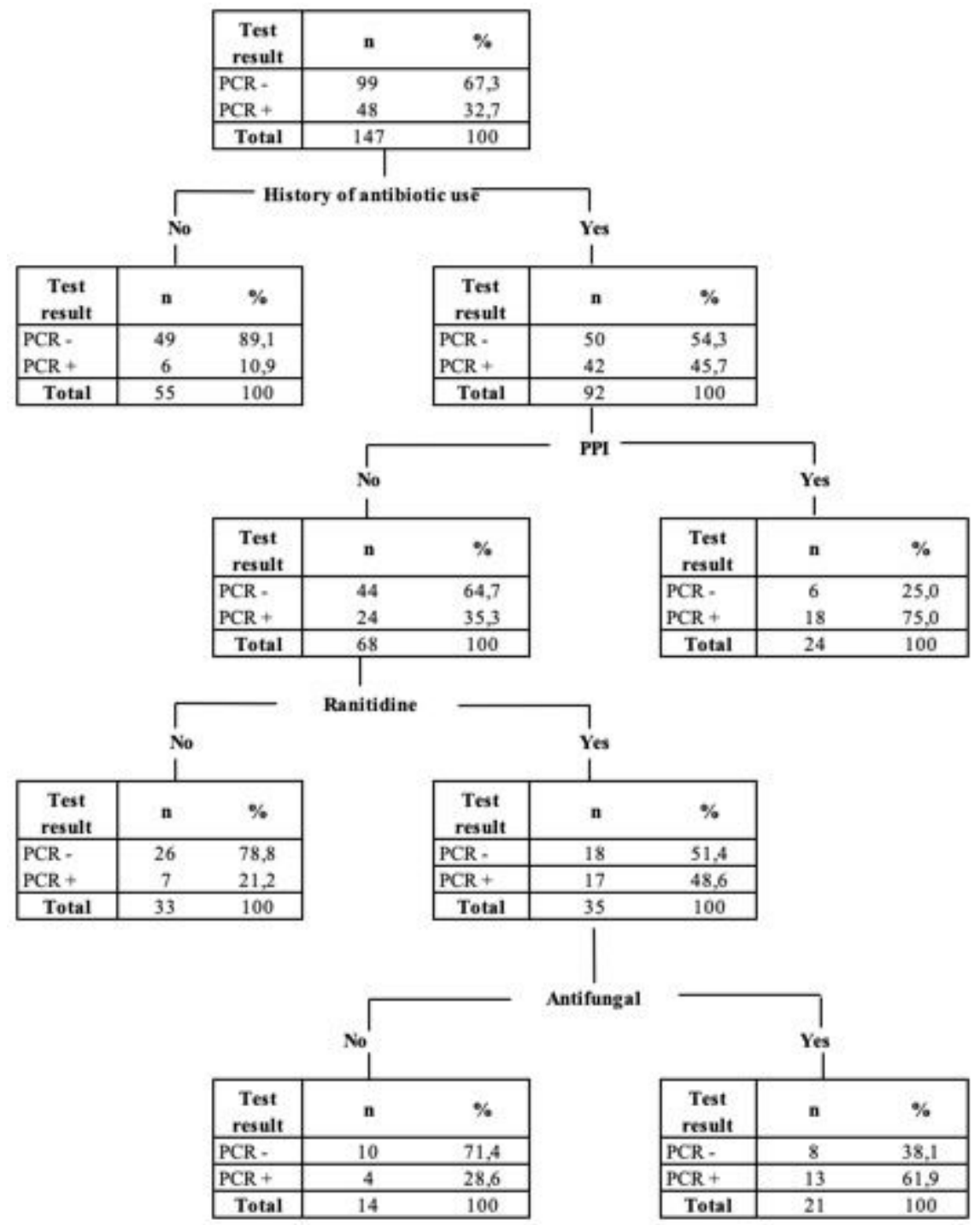

Figure 1

Classification tree for prediction CDI by PCR.

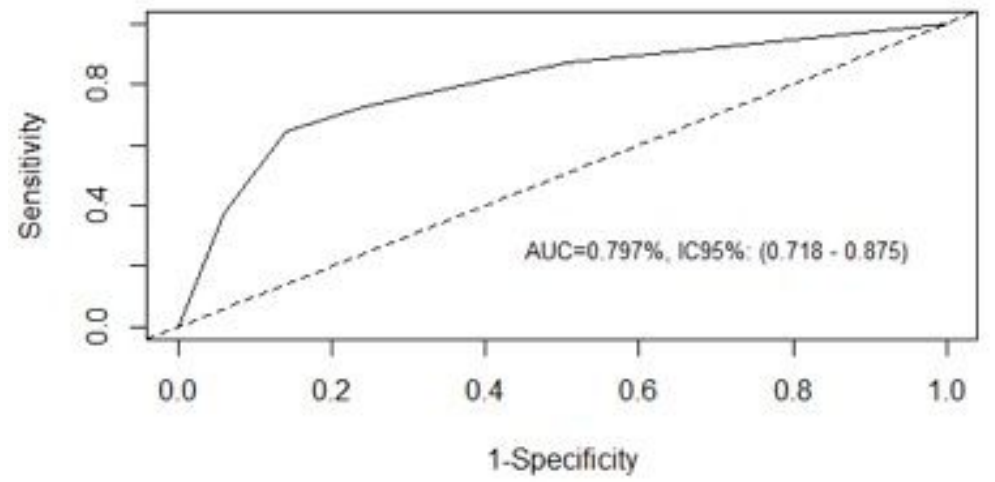


Figure 2

Results from validation of the classification tree. 